\title{
Common Factors Underlying the Five Facets of Mindfulness and Proposed Mechanisms: a Psychometric Study Among Meditators and Non-meditators
}

\author{
Kathrin Bednar ${ }^{1,2} \cdot$ Martin Voracek ${ }^{1} \cdot$ Ulrich S. $\operatorname{Tran}^{1}$ (D) \\ Published online: 2 September 2020 \\ (C) The Author(s) 2020
}

\begin{abstract}
Objectives This study investigated whether common factors underlie the established mindfulness facets, as assessed by the Five Facet Mindfulness Questionnaire (FFMQ) and some of the mechanisms, which have been previously proposed to explain the beneficial effects of mindfulness on mental health.

Methods Multigroup exploratory structural equation models (ESEM) were fitted to samples of non-meditators and meditators (total $N=3265$ ) to (1) identify the number of factors that underlie the facets and mechanisms of mindfulness, (2) establish measurement invariance, and (3) conduct path analyses to determine the associations of extracted factors with psychological symptoms.

Results Five measurement-invariant common factors were found to underlie the mechanisms and facets of mindfulness. The FFMQ facets loaded distinctly, but none of them highest, on these common factors. The common factors represented different ways of focusing, dealing with distress, and relating towards one's own thoughts, feelings, emotions, and body sensations. Three of the common factors appeared to specifically reflect meditation experience. The FFMQ facets accounted for less variance of depression, anxiety, somatization, and stress scores than marker scales of the five common factors, all of which derived from the proposed mechanisms.

Conclusions The common factors appear to be elements of the supporting mechanisms and psychological faculties of mindfulness. Their existence may explain the mutual interrelations between mechanisms and self-reported mindfulness but also suggests that self-reported mindfulness may not be factorially distinct from its assumed mechanisms. Longitudinal studies as well as behavioral data are needed to probe the generalizability and causality of these psychometric results.
\end{abstract}

Keywords Mindfulness facets $\cdot$ Mindfulness mechanisms $\cdot$ Exploratory structural equation model $\cdot$ Psychological symptoms

Mindfulness has recently received a lot of attention in scientific research and the media. Mindfulness is mostly referred to as the

Electronic supplementary material The online version of this article (https://doi.org/10.1007/s12671-020-01492-6) contains supplementary material, which is available to authorized users.

Ulrich S. Tran

ulrich.tran@univie.ac.at

1 Department of Cognition, Emotion, and Methods in Psychology, School of Psychology, University of Vienna, Liebiggasse 5, A-1010 Vienna, Austria

2 Institute for Information Systems and Society, Vienna University of Economics and Business, Vienna, Austria trait that can be trained with mindfulness exercises and meditation practice. According to Kabat-Zinn (Kabat-Zinn 2015, p. 1481), mindfulness "can be thought of as moment-to-moment, non-judgmental awareness, cultivated by paying attention in a specific way, that is, in the present moment, and as non-reactively, as non-judgmentally, and as openheartedly as possible". Most definitions of mindfulness (e.g., the two-component model of Bishop et al. 2004 with its respective factors self-regulated attention and orientation to experience) take up two characteristics mentioned here, namely, (1) paying attention to the present moment and (2) the nonjudging attitude of doing so.

Mindfulness is promising for its reported beneficial health effects, its negative association with psychological symptoms, and its positive influence on well-being in healthy as well as in clinical populations. Training mindfulness leads not only to higher self-reported mindfulness 
but also to the decreased symptoms of worry and depression (Delgado-Pastor et al. 2015). Mindfulness is reported to increase psychological well-being and have protective effects against the development of anxiety, depression, and other negative psychological symptoms (e.g., Keng et al. 2011). Even brief mindfulness programs can be successful at reducing symptoms of stress and increasing well-being (Greeson et al. 2014). While there is a large body of interventions based on mindfulness and meditation exercises, mindfulness-based cognitive therapy (MBCT; Segal et al. 2013) and mindfulness-based stress reduction (MBSR; Kabat-Zinn 2005) are standardized interventions with exact guidelines and protocols. Both of these mindfulness-based interventions improve symptoms of anxiety and depression (Hofmann et al. 2010; Khoury et al. 2013).

However, there is an ongoing debate among researchers regarding the construct of mindfulness (Van Dam et al. 2018). Different concepts, structures, underlying factors, and mechanisms of mindfulness have been proposed (for overviews, see Park et al. (2013) and Van Dam et al. 2018). Currently, mindfulness lacks a normative definition and there is no agreement as to which psychometric measures assess mindfulness adequately. Consequently, the boundaries of the construct of mindfulness appear not well defined. This creates ambiguity, as it thus remains undecidable whether mindfulness might be a very general construct with measurable effects even in the absence of formal or informal training (e.g., increases of mindfulness in non-mindfulness-based treatments; Goldberg et al. 2019), or whether this apparent generality is caused by an excessive overlap with other traits (e.g., with neuroticism and negative affect; Giluk 2009). Recent research highlights definitional overlaps of the trait-like (i.e., dispositional) aspects of self-reported mindfulness with the Big Five personality traits (especially with neuroticism and conscientiousness) and reported only small unique contributions of mindfulness in accounting for mental health, once the Big Five are statistically controlled for (Tran et al. 2020).

Researchers have thus called, in a sense, to go back to the start, by redirecting research towards the mental faculties, which support mindfulness, instead of attempting to measure mindfulness itself (Van Dam et al. 2018). This can be likened to intelligence research where particular cognitive capacities provide a conceptual framework for the construct of "intelligence."

Against this background, it can be argued that self-reported mindfulness likely contains useful information on how mindfulness exerts its effects (its supporting faculties). The following paragraphs review research targeting attention regulation, body awareness, emotion regulation, and change in perspective on the self (Hölzel et al. 2011; other mechanisms, which are presented, e.g., in the review of Gu et al. 2015, are outside the scope of the present study). These mechanisms have been previously proposed to explain the positive effects of mindfulness meditation on mental health (Hölzel et al. 2011) and of mindfulness-based interventions, such as MBSR and MBCT (Gu et al. 2015). Concerning change in the perspective on the self, decentering and nonattachment are discussedmechanisms that are part of two specific models of mindfulness (Brown et al. 2007; Shapiro et al. 2006). It appears tempting to ask whether these proposed mechanisms are not already among the defining elements of mindfulness as captured in popular self-report measures of mindfulness.

According to Kabat-Zinn (2015, p. 1481), mindfulness "basically boils down to paying attention". The ability to focus one's attention on internal and external stimuli is one crucial component of mindfulness and mindfulness meditation (Hölzel et al. 2011). Meditation practice leads to a more accurate, efficient, and flexible visual attention system (Hodgins and Adair 2010). While there is evidence of improved sustained, selective, and executive attention among long-term meditators, compared with novice meditators (Chiesa et al. 2011), short-term treatments like MBSR and MBCT appear to specifically benefit attention to mental representations only (Lao et al. 2016).

Mindfulness is the awareness of one's thoughts, emotions, and sensations (Brown et al. 2007). Mindfulness training that focuses on bodily sensations has additional positive effects on mental health and decreases physiological signals of stress (Delgado-Pastor et al. 2015). The awareness of body sensations therefore has been described as an important aspect of mindfulness. Mehling et al. (2011, p. 6) emphasize that "aspects of mindfulness appear to constitute a key element of body awareness". Mindfulness practice predicts higher body awareness (Quezada-Berumen et al. 2014). However, body awareness is often neglected in the various mindfulness measures.

Changes in emotional reactivity and momentary experiences of positive and negative affect (i.e., aspects of emotion regulation) are potential mechanisms of MBCT in the treatment of recurrent major depressive disorder (van der Velden et al. 2015). Increased activity of the hippocampus, a brain area associated with emotional memory and emotional processing, has been suggested to explain the beneficial effects of meditation on depression (Annells et al. 2016). Mindfulness and meditation may increase the exposure to emotional experiences and thus help to support the extinction of conditioned negative emotional reactions to stimuli (Hölzel et al. 2011). Top-down (cognitive control of emotional reactions and experiences; cognitive reappraisal) and bottom-up processes (absence of cognitive control; emotional nonappraisal) may be responsible for further effects of mindfulness on emotion regulation (Chiesa et al. 2013). Whereas cognitive control is increased in short-term mindfulness practitioners, it is 
decreased in long-term practitioners, who may perceive emotional stimuli in a more accepting way and feel less need for further control.

Decentering, or reperceiving, is a proposed metamechanism of mindfulness that helps to achieve a shift in perspective and facilitates the effects of other mechanisms of mindfulness (self-regulation; self-management; emotional, cognitive, and behavioral flexibility; values clarification; exposure; Shapiro et al. 2006). Decentering is the ability to "observe one's thoughts and feelings as temporary, objective events in the mind, as opposed to reflections of the self that are necessarily true" (Fresco et al. 2007, p. 234). It increases the "capacity for objectivity in relationship to one's internal/external experience", thereby allowing to "dis-identify from thoughts, emotions, and body sensations as they arise, and simply be with them instead of being defined (i.e., controlled, conditioned, determined) by them" (Shapiro et al. 2006, p. 378). Decentering is considered a core mechanism for dealing with stress (Creswell and Lindsay 2014) and might play an essential role for the effects of MBCTs (Bieling et al. 2012).

Nonattachment is a Buddhist concept, with similarities to decentering. It refers to an "acceptance of, or willingness to be with, what is" (Brown et al. 2007, p. 227). Nonattachment is related to the Western concept of secure attachment where "the nonattached individual genuinely cares about, is engaged in, and responsive to the present situation without falling into self-aggrandizement or self-degradation" (Sahdra et al. 2010, p. 118) and is promoted by a wide range of practices (contemplative, spiritual, artistic, psychotherapeutic, or mundane) if they involve a "letting go" of fixations. Nonattachment correlates positively with psychometric mindfulness and negatively with depression, anxiety, stress, difficulties in emotion regulation, and suicide rumination (Lamis and Dvorak 2014; Sahdra et al. 2010).

Currently, mindfulness research tries to explain how mindfulness exerts its positive effects on mental health, testing whether specific constructs, like the ones described above, mediate the effects of mindfulness on mental health (for a review and meta-analysis of longitudinal studies, see $\mathrm{Gu}$ et al. 2015; for recent cross-sectional studies, see Burzler et al. 2019; Freudenthaler et al. 2017; Sahdra et al. 2016; Tran et al. 2014). However, longitudinal studies (see Gu et al. 2015) also suggest that self-reported mindfulness is a mechanism of change for mindfulness treatments (and probably for other, non-mindfulness-based, treatments as well, see Goldberg et al. 2019). Thus, popular mindfulness measures likely already capture some of the "mental faculties" of mindfulness (sensu Van Dam et al. 2018). To our knowledge, no previous study has systematically tested whether mindfulnessrelated constructs, which have been proposed as mechanisms in theoretical models, are factorially distinct (i.e., are not explained by the same underlying factors) from self-report measures of mindfulness. Research may thus have skipped an important step so far.

In order to be a mediating variable, a mechanism needs to correlate with mindfulness and be an independent construct. Otherwise, the observation, or claim, that a mechanism explains the effects of mindfulness would be tautologous. Yet, if a mechanism and mindfulness arise from the same underlying factors, they may not be conceptually independent. Pursuing a similar goal, Siegling and Petrides (2016) have examined the extent other mindfulness scales add to the construct variance as accounted for by one specific self-report measure of mindfulness, the Five Facet Mindfulness Questionnaire (FFMQ; Baer et al. 2006). They reported a large overlap which indicates that the investigated instruments mostly measure the same construct. Closing a similar research gap concerning some of the proposed mechanisms of mindfulness may help to clarify the boundaries of self-reported mindfulness but also its very concept.

The FFMQ purports five facets of mindfulness and has been derived from a number of equally widely used alternative measures. It can thus be considered comprehensive. The FFMQ shares four of its five facets with one of its predecessors, the Kentucky Inventory of Mindfulness Skills (Baer et al. 2004). These facets were originally devised as the "what" and "how" skills of mindfulness in dialectical behavior therapy (e.g., Linehan 2015). Thus, the instrument bears a strong connection to one theoretical model of mindfulness. Also, psychometric studies have replicably shown that the higher-order structure of the FFMQ agrees well with the two-component model of mindfulness that was proposed by Bishop et al. (2004), both in meditating (Tran et al. 2014) and nonmeditating samples (Burzler et al. 2019; Tran et al. 2013). Being both comprehensive and compatible with theoretical models of mindfulness, the FFMQ appears thus to be a viable starting point for deeper analyses of self-reported mindfulness. At the same time, the structure of self-reported mindfulness, as measured with the FFMQ, differs between meditators and non-meditators, as do its associations with mental health (e.g., Baer et al. 2006; Tran et al. 2013, 2014). Comparing meditators and non-meditators in analysis may thus provide further important insights.

We present psychometric data on the common latent structure of mindfulness, as assessed with the FFMQ, and the above outlined constructs of attention regulation, body awareness, emotion regulation, decentering, and nonattachment. We also present results on the incremental validity of the common factors of mindfulness and its proposed mechanisms in accounting for mental health (depression, anxiety, somatization, and perceived stress). All analyses differentiated between regular meditators and non-meditators, testing the common factors also for measurement equivalence. 


\section{Method}

\section{Participants}

This study utilized previously published data (Burzler et al. 2019; Freudenthaler et al. 2017) and novel data. The pooled sample comprised in total $N=3265$ participants (53\% women) from the general population, with ages ranging from 18 to 87 years $(M=34.38, S D=15.27)$. The sample in majority included Austrian (51\%) and German (43\%) nationals, $6 \%$ indicated other (mostly central-European) nationalities (31 missing values). Half (50\%) of the sample had completed upper secondary education, $25 \%$ lower secondary education, and $24 \%$ had a university diploma (30 missing values). Sixtytwo percent indicated that they were in a relationship or married (single, $32 \%$; other, $6 \% ; 25$ missing values).

The sample included in total $n=408(12.5 \%)$ individuals who indicated that they practiced meditation or mindfulness exercises on a regular basis (i.e., at least once a week). These participants were classified as meditators in the present study. Everyone else was classified as non-meditator $(n=2857$, $87.5 \%)$. On average, meditators were slightly older than non-meditators $(M=37.35, S D=15.66$ vs. $M=33.95, S D=$ $15.17 ; d=0.22, p<.001)$ and were with higher proportion female $(63.5 \%$ vs. $51.5 \% ; p<.001)$. Meditators reported on average 3 years of regular meditation practice $(M d n=3$, interquartile range $=1-8$ ), whereof $63.2 \%$ had 1 to 10 years, $19.6 \%$ more than 10 years (ranging up to 40 years), and $3.9 \%$ less than 1 year of meditation experience (54 missing values). Meditators practiced mostly yoga (50\%), followed by Zen (11\%), Qi Gong (6\%), mindfulness meditation (4\%), Transcendental Meditation (4\%), and Tai Chi (3\%) (other forms of meditation had prevalence rates $\leq 2 \%, 14 \%$ reported idiosyncratic styles; based on the data of 362 persons).

\section{Procedures}

Data collection was crowdsourced and distributed to a number of research assistants to minimize recruitment bias and to increase sample heterogeneity. Participation was voluntary, anonymous, and unremunerated. Participants gave their informed consent to participate in the study. The overall sample comprised students and individuals from the general population. Survey forms were filled in individually and in quiet facilities. Participants had to be 18 years of age or older and fluent in German (the survey language). Otherwise, there were no exclusion criteria.

\section{Measures}

Participants provided sociodemographic data and filled in the inventories described below. Detailed group comparisons of mean scores and measures of internal consistency (Revelle's omega total, using the $\mathrm{R}$ package psych for computations; Revelle 2008) in the current study are provided in Supplemental Materials. German forms of the SBC and NAS were created with the parallel blind technique (Behling and Law 2000).

Meditation Experience Participants were asked about the frequency of practice of (1) meditation and mindfulness techniques, (2) autogenic training or progressive muscle relaxation, and (3) other relaxation techniques. Participants could choose between never, not regularly, and regular practicing (once, twice, three times, and (more than) four times per week). Additionally, participants estimated the daily time they spent practicing (in minutes) and the number of years for which they had been practicing.

Mindfulness Mindfulness was measured with the 39-item Five Facet Mindfulness Questionnaire (FFMQ; German form: Tran et al. 2013). The FFMQ comprises five facets: Observing (e.g., "I pay attention to sensations, such as the wind in my hair or sun on my face"), Describing (e.g., "I'm good at finding the words to describe my feelings"), Acting with Awareness (e.g., "When I do things, my mind wanders off and I'm easily distracted", reverse-scored), Nonjudging of inner experience (e.g., "I make judgments about whether my thoughts are good or bad", reversescored), and Nonreactivity to inner experience (e.g., "I perceive my feelings and emotions without having to react to them"). Observing and Describing capture "what" skills (what one does when practicing mindfulness), Nonjudging a "how" skill, and Acting with Awareness captures aspects of both "what" and "how" skills (being focused on, and involved in, in one activity at a time, without losing focus; see Baer et al. 2004). Eight items load on each of the facets, except for Nonreactivity, which comprises seven items. Participants indicate how often statements apply on a 5 -point response scale $(1=$ never true, $5=$ very often true).

Attention Regulation The Effortful Control (EC) scale of the Adult Temperament Questionnaire (ATQ; German form: Wiltink et al. 2006) was used to measure the ability to focus attention. The EC scale has three subscales: Attentional Control, which refers to the capacity to shift and focus attention (5 items); Inhibitory Control, which describes the capacity to inhibit inappropriate approach behavior and to suppress positively toned impulses (7 items); and Activation Control, which characterizes the capacity to perform an action and to suppress negatively toned impulses (7 items). Responses are given on a 7 point Likert scale $(1=I$ do not agree at all, $4=$ neutral, $7=$ I fully agree $)$. 
Body Awareness The Scale of Body Connection (SBC; Price and Thompson 2007) was used to assess body awareness. It comprises two subscales, Body Awareness (12 items, e.g., "I am aware of tension in my body") and Body Dissociation ( 8 items, e.g., "I find it difficult to identify my emotions"). The 20 items are assessed on a 5 -point response scale $(0=$ not true at all, $4=$ always true). All items of the Body Dissociation subscale were reverse-scored to attain a positive interpretation, henceforth termed Body Association.

Emotion Regulation Aspects of emotion regulation were assessed with the Difficulties in Emotion Regulation Scale (DERS; German form: Ehring et al. 2008). The DERS comprises 36 items with six subscales, Nonacceptance of Emotional Responses (6 items), Difficulties Engaging in Goal-Directed Behavior (5 items), Impulse Control Difficulties (6 items), Lack of Emotional Awareness (6 items), Limited Access to Emotion Regulation Strategies (8 items), and Lack of Emotional Clarity (5 items). Gratz and Roemer (2004) reported good psychometric properties of the DERS in student samples, including a good test-retest reliability, and adequate construct and predictive validity. The DERS items are rated on a 5 -point Likert scale $(1=$ almost never, $5=$ almost always). All scales were reverse-scored in the present study so that higher scores indicated greater emotion regulation abilities instead of greater difficulties and scale names were changed accordingly (see Tran et al. 2014).

Decentering The German form of the Experiences Questionnaire Decentering scale (EQ-D; Gecht et al. 2014) was used to assess two subdimensions of decentering, as suggested by Gecht et al. (2014): Accepting Self-Perception (4 items, e.g., "I am better able to accept myself as I am") and Distanced Perspective (4 items, e.g., "I can separate myself from my thoughts and feelings"). Responses are given on a 5point scale $(1=$ never, $5=$ always $)$.

Nonattachment The Nonattachment Scale (NAS; Sahdra et al. 2010) was used to assess nonattachment. The NAS comprises 30 items (e.g., "I can enjoy the pleasures of life without feeling sad or frustrated when they end"), which are rated on a 6-point response scale $(1=$ I do not agree at all, $6=$ I fully agree $)$ and yield a total score.

Anxiety, Depression, and Somatization The German form of the 18-item Brief Symptoms Inventory (BSI-18; Franke 2016) was used to assess symptoms of anxiety (5 items), depression (6 items), and somatization (7 items). Participants indicated whether, and if so, to what extent, they suffered from the described symptoms in the past week on a 5-point Likert scale $(0=$ not at all, $4=$ extremely $)$.
Perceived Stress Perceived stress was assessed with the German form of the Perceived Stress Questionnaire (PSQ; Fliege et al. 2005), which is composed of 20 items and provides measures of four factors of perceived stress (worries, tension, joy, demands) and a total score that was used in the present study. Participants rate how often they have experienced the described symptoms in the past 4 weeks on a 4-point response scale $(1=$ almost never, $4=$ usually $)$.

\section{Data Analyses}

In a first step, a multigroup exploratory structural equation modeling (ESEM; Marsh et al. 2014) was carried out to identify the number of common factors that represented all mindfulness facets and mechanisms on their scale and subscale levels in the two groups. ESEM allows for the free estimation of cross-loadings, like exploratory factor analysis, but also for the correction of measurement error, multigroup modeling, and the simultaneous analysis of relations between latent constructs and other constructs. It is specifically suited for the exploration of complex factor structures, as expected here, and has been previously shown to also better capture well-defined factor structures, like of the Big Five of personality (see Booth and Hughes 2014), than conventional confirmatory factor analysis (CFA). Like CFA, ESEM reports modification and goodness-of-fit indices. Therefore, factor analyses were conducted with multigroup ESEM.

Factorial/measurement invariance on the scale and subscale level was then tested systematically, starting with configural and weak measurement invariance and moving on to strong and strict measurement invariance as suggested in the taxonomy of Marsh et al. (2014); see Supplemental Materials. Each model represents a higher level of invariance with an increasing number of parameters constrained to be equal across groups. Once the model that represented the most adequate level of measurement invariance was identified, a multigroup regression analysis was conducted to investigate the associations of the extracted factors with perceived stress, depression, anxiety, and somatization. This model included the extracted factors of the ESEM model as latent variables and, hence, accounted for measurement errors in the independent variables.

Analyses were performed with Mplus 8.4 (Muthén and Muthén 1998-2017). Full-information maximum likelihood (FIML) was used to estimate the parameters based on all available data. Of the 3265 participants, $95.4 \%$ had less than three missing values across all items. Standard errors were estimated with robust maximum likelihood (MLR estimator in Mplus), which corrects for non-normal distributions in the endogenous variables. For the ESEM analysis, GEOMIN rotation was used; standardized parameter estimates are reported throughout. 
Assessment of Model Fit We report $\chi^{2}$ tests of model fit as well as the values of several fit indices for model evaluations and comparisons: The comparative fit index (CFI), the Tucker-Lewis index (TLI), and the root mean square error of approximation (RMSEA) with its $90 \%$ confidence interval. In the present study, we utilized cutoffs for "acceptable" and "good" model fits as devised by $\mathrm{Hu}$ and Bentler (1999): <0.08/<0.06 (RMSEA) and > 0.90/> 0.95 (CFI/TLI), respectively.

Concerning tests of measurement invariance, we present $\Delta \chi^{2}$ test statistics on the difference between more liberal and more strict models as well as the difference in CFI $(\triangle \mathrm{CFI})$, using a cutoff of $<-0.002$ to decide whether a stricter model leads to a notable decrease in model fit (see Putnick and Bornstein 2016). We preferentially interpreted the difference in CFI values, rather than the significance of the $\Delta \chi^{2}$ test, in cases where these two indicators provided contradictive results, as the $\Delta \chi^{2}$ test is known to be sensitive to sample size.

To decide for an adequate number of common factors in the ESEM analysis, fit indices were assessed with consideration of strict cutoffs for CFI, TLI, RMSEA, and the overlap of RMSEA confidence intervals. Furthermore, $\triangle$ CFI was evaluated in the opposite direction, compared with its use in tests of measurement invariance: Instead of a decrease in model fit of $<-0.002$, an increase of $>+0.01$ was taken into consideration as a criterion for model comparisons. Where modification indices (MI) showed values higher than 20, constrained parameters were estimated freely when it could be justified on theoretical grounds.

\section{Results}

\section{Number of Common Factors}

The results of the multigroup ESEM analyses, testing one- to six-factor models, indicated the best fit for a modified 5-factor model, as displayed in Table 1. Modification indices of the 5factor ESEM suggested a substantial increase in model fit when the residuals of the FFMQ facet Nonjudging and the DERS subscale Acceptance of Emotional Responses were allowed to correlate. Both scales include items that express a critical attitude towards one's own thoughts and feelings (e.g., "When I'm upset, I become embarrassed for feeling that way" [DERS]; "I criticize myself for having irrational or inappropriate emotions" [FFMQ]). Correlated residuals for these two scales therefore were included in the 5-factor model. Compared with this modified 5-factor model, adding a sixth factor did not substantially increase model fit $(\Delta \mathrm{CFI}=+$ 0.007). Furthermore, the RMSEA confidence intervals of the modified 5-factor model and the 6-factor model overlapped. Thus, the more parsimonious modified 5-factor model was retained as the final model.

\section{Tests of Measurement Invariance}

Six models representing different levels of factorial/ measurement invariance on the scale and subscale level of the investigated measures between regular meditators and non-meditators were compared for the modified 5-factor model (see Table 1). Descriptively, most invariance models showed an acceptable data fit. However, considering the

Table 1 Model fit statistics of the ESEM analyses and the tests of measurement invariance

\begin{tabular}{|c|c|c|c|c|c|c|}
\hline Model & $\chi^{2}(d f)$ & CFI & TLI & RMSEA [90\% CI] & $\Delta \mathrm{CFI}$ & $\Delta \chi^{2}(d f)$ \\
\hline \multicolumn{7}{|c|}{ Number of ESEM factors } \\
\hline 1 factor & 8463.07 (304) & 0.662 & 0.620 & $0.128[0.126,0.131]$ & & \\
\hline 2 factors & $4761.74(268)$ & 0.814 & 0.763 & $0.101[0.099,0.104]$ & +0.152 & \\
\hline 3 factors & $3368.92(234)$ & 0.870 & 0.810 & $0.091[0.088,0.093]$ & +0.056 & \\
\hline 4 factors & $1863.13(202)$ & 0.931 & 0.884 & $0.071[0.068,0.074]$ & +0.061 & \\
\hline 5 factors & $1029.08(172)$ & 0.965 & 0.929 & $0.055[0.052,0.059]$ & +0.034 & \\
\hline 5 factors modified & 763.15 (170) & 0.975 & 0.951 & $0.046[0.043,0.050]$ & +0.010 & \\
\hline 6 factors & $578.03(144)$ & 0.982 & 0.957 & $0.043[0.039,0.047]$ & +0.007 & \\
\hline \multicolumn{7}{|c|}{ Tests of measurement invariance } \\
\hline Model 0 & $763.15(170)$ & 0.975 & 0.951 & $0.046[0.043,0.050]$ & & \\
\hline Model 1 & $824.40(240)$ & 0.976 & 0.966 & $0.039[0.036,0.042]$ & +0.001 & $88.03(70)$ \\
\hline Model 2 & $868.12(254)$ & 0.975 & 0.966 & $0.038[0.036,0.041]$ & -0.001 & $43.36(14)^{* * *}$ \\
\hline Model 3 & $891.60(269)$ & 0.974 & 0.967 & $0.038[0.035,0.040]$ & -0.001 & $26.00(15)^{*}$ \\
\hline Model 4 & $1093.98(274)$ & 0.966 & 0.958 & $0.043[0.040,0.045]$ & -0.008 & $223.19(5)^{* * *}$ \\
\hline
\end{tabular}

CFI comparative fit index, TLI Tucker-Lewis index, RMSEA root mean square error of approximation, $C I$ confidence interval, $\triangle C F I$ change in CFI. Boldface indicates the final models. In order to enable the evaluation of the fit of the different models with stated relative cutoff values (see main text), three digits are presented for CLI, TLI, RMSEA, and $\Delta$ CFI values. For all $\chi^{2}$ tests of model fit, $p<0.001$. Otherwise, ${ }^{*} p<0.05, * * * p<0.001$ 
Table 2 Factor loadings in the modified 5-factor strict measurement invariance model

\section{Factor}

\begin{tabular}{|c|c|c|c|c|c|c|}
\hline Scales and Subscales & 1 & 2 & 3 & 4 & 5 & $h^{2}$ \\
\hline FFMQ Observing & $.02 / .02$ & $.68 / .72$ & $.00 / .00$ & $.00 / .00$ & $-.03 /-.03$ & $.45 / .51$ \\
\hline FFMQ Describing & $.09 / .10$ & $.19 / .20$ & $.02 / .02$ & $-.07 /-.08$ & $.57 / .58$ & $.45 / .48$ \\
\hline FFMQ Acting with Awareness & $.49 / .51$ & $-.01 /-.01$ & $.07 / .07$ & $.02 / .02$ & $.27 / .28$ & $.48 / .52$ \\
\hline FFMQ Nonjudging & $-.02 /-.02$ & $-.13 /-.13$ & $.18 / .18$ & $.28 / .29$ & $.34 / .34$ & $.38 / .39$ \\
\hline FFMQ Nonreactivity & $.02 / .02$ & $.18 / .19$ & $.69 / .72$ & $.06 / .06$ & $-.05 /-.05$ & $.57 / .61$ \\
\hline NAS Nonattachment & $.10 / .10$ & $.21 / .21$ & $.34 / .34$ & $.30 / .29$ & $.12 / .12$ & $.55 / .54$ \\
\hline SBC Body Awareness & $.03 / .03$ & $.77 / .81$ & $-.07 /-.08$ & $.04 / .04$ & $-.01 /-.01$ & $.58 / .64$ \\
\hline SBC Body Association & $.06 / .05$ & $-.13 /-.12$ & $-.05 /-.05$ & $.16 / .15$ & $.72 / .69$ & $.62 / .56$ \\
\hline EQ Accepting Self-Perception & $-.04 /-.04$ & $.28 / .30$ & $.28 / .30$ & $.22 / .23$ & $.23 / .25$ & $.45 / .51$ \\
\hline EQ Distanced Perspective & $.06 / .06$ & $-.12 /-.11$ & $.77 / .76$ & $.08 / .07$ & $.01 / .01$ & $.73 / .71$ \\
\hline EC Attentional Control & $.82 / .81$ & $-.01 /-.01$ & $.08 / .08$ & $-.02 /-.02$ & $.02 / .02$ & $.74 / .72$ \\
\hline EC Inhibitory Control & $.36 / .37$ & $.01 / .01$ & $.28 / .29$ & $-.06 /-.06$ & $.00 / .00$ & $.27 / .29$ \\
\hline EC Activation Control & $.58 / .58$ & $.04 / .04$ & $-.07 /-.07$ & $.11 / .11$ & $.06 / .06$ & $.41 / .41$ \\
\hline DERS Acceptance of Emotion & $.00 / .00$ & $-.04 /-.04$ & $.03 / .03$ & $.60 / .64$ & $.19 / .20$ & $.52 / .58$ \\
\hline DERS Goal-directed Behavior & $.57 / .57$ & $-.03 /-.03$ & $-.01 /-.01$ & $.43 / .43$ & $-.12 /-.12$ & $.67 / .67$ \\
\hline DERS Impulse Control & $.13 / .13$ & $-.01 /-.01$ & $.08 / .08$ & $.59 / .62$ & $.05 / .06$ & $.55 / .60$ \\
\hline DERS Emotional Awareness & $-.05 /-.05$ & $.55 / .57$ & $.03 / .03$ & $-.06 /-.06$ & $.36 / .38$ & $.54 / .59$ \\
\hline DERS Emotion Regulation Strategies & $.02 / .02$ & $.06 / .06$ & $.06 / .06$ & $.86 / .90$ & $.00 / .00$ & $.82 / .90$ \\
\hline DERS Emotional Clarity & $.06 / .06$ & $.17 / .18$ & $-.05 /-.05$ & $.21 / .22$ & $.56 / .61$ & $.56 / .65$ \\
\hline
\end{tabular}

Factor determinacies

$.92 / .92$

$.89 / .90$

$.91 / .91$

$.94 / .96$

$.89 / .90$

$h$ communality, FFMQ Five Facet Mindfulness Questionnaire, $N A S$ Nonattachment Scale, SBC Scale of Body Connection, $E Q$ Experiences Questionnaire, EC Effortful Control, DERS Difficulties in Emotion Regulation; factor determinacies = validity coefficients, which indicate the correlation between factor score estimates and their corresponding factors. Standardized factor loadings are presented for non-meditators (left) and meditators (right); in the investigated model, unstandardized factor loadings were set to equality; due to differences in dispersion, standardized loadings may differ between groups. Factor loadings $>0.30$ in both samples are set in boldface, and the highest loading in each factor is highlighted by shading. Not significant $(p>.05)$ loadings are set in italics 
Table 3 Factor intercorrelations

\begin{tabular}{lllll}
\hline Factor & 1 & 2 & 3 & 4 \\
\hline 2 & 0.05 & & & \\
3 & $0.50^{* * *}$ & $0.08^{* *}$ & & \\
4 & $0.50^{* * *}$ & 0.00 & $0.60^{* * *}$ & \\
5 & $0.36 * * *$ & $0.32^{* * *}$ & $0.29 * * *$ & $0.42^{* * * *}$ \\
\hline
\end{tabular}

Factor intercorrelations were equal among non-meditators and meditators as latent factor covariances were set to equality in the invariance model. $* * p<0.01, * * * p<0.001$

results of the $\Delta \chi^{2}$ tests and the $\Delta$ CFI values, Model 3 (strict measurement invariance: equal factor loadings, equal intercepts, and equal factor covariances) appeared to provide the best fit.

Factor loadings for regular meditators and nonmeditators are displayed in Table 2 and factor intercorrelations in Table 3 (see Supplemental Materials for Mplus syntax). Most factor intercorrelations were of medium to large size, with coefficients ranging from .28 to .58 (see Table 3). The highest intercorrelation was observed between Factors 3 and 4.

Factor 1 subsumed with highest loadings Attentional Control, Acting with Awareness, Goal-Directed Behavior, and Activation Control. All of these scales comprise items that express the ability to stay focused on a planned activity despite distractions or the experience of negative emotions ("how" skills).

Factor 2 subsumed with highest loadings Body Awareness, Observing, and Emotional Awareness: scales that measure awareness of feelings and bodily sensations ("what" skills).

Factor 3 subsumed only two scales with high loadings: The Decentering subscale Distanced Perspective and Nonreactivity. Both scales comprise items that suggest a distance or separation from thoughts, feelings, and emotions and to be able to "step back" without the need for immediate reaction ("how" skills).

Factor 4 represented five out of the six DERS subscales, with Emotion Regulation Strategies loading highest, followed by Acceptance of Emotional Responses, Impulse Control, and Goal-directed Behavior. Items of these four DERS scales assess the ability to deal with, and accept, feelings and emotions and control one's behavior ("how" skills).

Factor 5 subsumed with the highest loadings Body Association, Emotional Clarity, and Describing. These three scales assess the ability to identify and accurately describe one's feelings and emotions ("what" skills).

Notably, none of the FFMQ facets loaded highest on any of the common factors. However, all facets, except Nonjudging, loaded distinctively $(>0.30)$ on only one of the factors. Nonjudging, Nonattachment, Accepting Self-Perception, and Inhibitory Control had only weak factor loadings each (ranging between 0.20 and 0.40 ) and loaded diffusely on more than one factor. Nonjudging loaded on Factors 4 and 5, and Inhibitory Control loaded on Factors 1 and 3. The factor loadings of Nonattachment were distributed over Factors 2 to 4 and of the Decentering subscale Accepting Self-Perception over Factors 2 to 5.

Compared with non-meditators, regular meditators showed significantly higher means in all common factors. Differences were largest in Factor $2(d=0.84, p<0.001)$ and Factor $3(d=$ $0.38, p<0.001$ ), but smaller in the remaining factors (Factor $1: d=0.13, p=0.008$; Factor 4: $d=0.18, p<.001$; Factor 5: $d=0.17, p=0.001)$.

\section{Associations with Psychological Symptoms and Perceived Stress}

The final measurement invariance model was combined with a path model to test the associations of the common factors with mental health. The model had a good fit, $\chi^{2}(381)=$

Table 4 Standardized path coefficients of the common factors to psychological symptoms and stress

\begin{tabular}{|c|c|c|c|c|c|c|c|c|}
\hline \multirow[t]{2}{*}{ Outcome } & \multicolumn{5}{|l|}{ Factor } & \multicolumn{3}{|c|}{ Variance accounted for by } \\
\hline & 1 & 2 & 3 & 4 & 5 & $\begin{array}{l}\text { Common } \\
\text { factors }\end{array}$ & $\begin{array}{l}\text { Marker } \\
\text { scales } \\
\text { only }\end{array}$ & $\begin{array}{l}\text { FFMQ } \\
\text { facets } \\
\text { only }\end{array}$ \\
\hline Depression & $0.05 * /-0.03$ & $0.04 / 0.10^{*}$ & $-0.03 /-0.16^{*}$ & $-0.58 * * * /-0.40 * * *$ & $-0.20 * * * /-0.18 * *$ & $45 \% / 38 \%$ & $39 \% / 30 \%$ & $26 \% / 24 \%$ \\
\hline Anxiety & $-0.05 /-0.14$ & $0.16^{* * *} / .07$ & $-0.10 * * /-0.23 * * *$ & $-0.34 * * * /-0.29 * * *$ & $-0.23 * * * /-0.03$ & $32 \% / 31 \%$ & $27 \% / 25 \%$ & $20 \% / 18 \%$ \\
\hline Somatization & $-0.02 / 0.00$ & $0.20^{* * / 0.19 * *}$ & $-0.06 /-0.28^{* * *}$ & $-0.26^{* * * /-0.19^{*}}$ & $-0.26^{* * * /-0.18 *}$ & $23 \% / 27 \%$ & $19 \% / 20 \%$ & $13 \% / 13 \%$ \\
\hline $\begin{array}{l}\text { Perceived } \\
\text { stress }\end{array}$ & $-0.07 * * /-0.08$ & $0.02 /-0.04$ & $-0.20 * * * /-0.32 * * *$ & $-0.32 * * * /-0.20 * * *$ & $-0.21 * * * /-0.27 * * *$ & $39 \% / 46 \%$ & $34 \% / 39 \%$ & $28 \% / 34 \%$ \\
\hline
\end{tabular}

Common factors = multigroup ESEM model, which included all scales and subscales of mindfulness and the proposed mechanisms; marker scales only = multigroup path model, which included only the highest loading scales of the five common factors (EC Attentional Control, SBC Body Awareness, EQ Distanced Perspective, DERS Emotion Regulation Strategies, SBC Body Association) as predictors; FFMQ facets only = multigroup path model, which included only the five FFMQ facets as predictors. Coefficients are presented for the full model (standardized path coefficients) and for non-meditators (left) and meditators (right) 
$1368.31, p<0.001, \mathrm{CFI}=0.968, \mathrm{TLI}=0.958$, RMSEA $=0.040[0.038 ; 0.042]$. Table 4 displays the standardized path coefficients and the variance of psychological symptoms and perceived stress accounted for by the common factors.

Overall, Factor 4 was associated the most with psychological symptoms and perceived stress, Factor 1 the least; Factor 3 appeared more important among regular meditators than non-meditators and Factor 5 more important among nonmeditators than regular meditators. Whereas the associations of the common factors with the outcomes were mostly negative, where significant, the associations of Factor 2 with anxiety and somatization were positive in both groups. Overall, the factors accounted for 23 to $46 \%$ of the outcome variance. The most variance was accounted for depression and perceived stress, the least for somatization.

Two further multigroup path models were fitted on the data: in the first model, only the marker (highest loading) scales (EC Attentional Control, SBC Body Awareness, EQ Distanced Perspective, DERS Emotion Regulation Strategies, SBC Body Association) of the common factors were used as predictors for the mental health outcomes; in the second model, only the five FFMQ subscales were used. The results are presented in Table 4. Overall, the marker scales accounted for less outcome variance than the five common factors. However, in direct comparison, they accounted for more variance than the FFMQ facets on their own.

\section{Discussion}

This psychometric study set out to examine whether some of the proposed mechanisms of mindfulness might be among the defining elements of the construct, as assessed in self-report, investigating samples of both regular meditators and nonmeditators alike. We found that five common factors underlay the facets of mindfulness, as measured with the FFMQ, and measures of attention regulation, body awareness, emotion regulation, decentering, and nonattachment. This suggests that the FFMQ and some of the proposed mechanisms of mindfulness are not factorially distinct, but arise from the same underlying factors, which we perceive as elements of the supporting mechanisms and faculties of mindfulness. Thus, one may argue that the FFMQ is not a mere measure of mindfulness, but already of some of its supporting mechanisms and mental faculties, which may explain the overlap of self-reported mindfulness with constructs like neuroticism and the apparent gains of mindfulness in non-mindfulness-based therapies. Yet, the results suggest further that more specific alternative measures might better capture these mechanisms/faculties of mindfulness than the FFMQ.

The five common factors covered attentional control (Factor 1), body awareness (Factor 2), decentering (Factor
3), emotion regulation (Factor 4), and the ability to identify/ describe feelings and emotions (Factor 5). Three of the common factors (Factors 3 to 5) covered different aspects of emotion regulation. This highlights the known overall importance of emotion regulation for the construct of mindfulness (e.g., Chiesa et al. 2013; Hölzel et al. 2011). At the same time, this also shows that emotion regulation is not a unitary construct within mindfulness, but appears to be split into distinct "what" and "how" skills.

\section{Decentering and Emotion Regulation}

Decentering (Factor 3), is one of the two factors (the other being Factor 2), which appeared to a relevant extent higher among regular meditators than non-meditators in the current study. As further suggested by the current results, decentering is also a mechanism, which unfolds its associations with mental health only among those with regular meditation experience. This result is corroborated by previous findings, which suggest an altered, more important, role of Nonreactivity (which also loads on this factor) for mental health among regular meditators, compared with non-meditators (Tran et al. 2013, 2014), and that Nonreactivity is a mechanism of change of meditation (Gao et al. 2018). Decentering (Factor 3) thus appears to be a distinct "how" mechanism/faculty of mindfulness, which increases through meditation experience. This adds to the evidence that decentering may be an important and defining element of mindfulness (Shapiro et al. 2006) and one specific factor that may play a role for stress regulation (Creswell and Lindsay 2014) and for the efficacy of mindfulness interventions (Bieling et al. 2012). The current findings further corroborate that decentering, as measured with the EQ, is no unitary construct and that subscale scores should be used in research (Gecht et al. 2014).

In contrast, the other two factors (Factors 4 and 5), which covered further aspects of emotion regulation, including an accepting attitude ("how") and the ability to describe and identify emotions ("what"), appeared to be more general. Both factors were broadly and similarly associated with mental health among regular meditators and non-meditators alike, and mean differences between regular meditators and nonmeditators were only small. Previous research has highlighted the overall importance of emotion regulation for mindfulness. However, the current results suggest that Factors 4 and 5 may also be responsible for (part of) the overlap of the mindfulness construct with constructs like neuroticism (Giluk 2009) and increases of mindfulness in non-mindfulness-based treatments (Goldberg et al. 2019): both factors were highly relevant for mental health (highest associations with mental health in the current study), but—lacking obvious differences between regular mediators and non-meditators - apparently not very specific for meditation experience. 
Emotion regulation accounts for most of the associations of dispositional mindfulness with mental health among the general population (Burzler et al. 2019; Freudenthaler et al. 2017). However, emotion regulation also exhibits close links with neuroticism (for a meta-analysis of neuroimaging studies, see Servaas et al. 2013), which is an important predictor of mental health on its own. Spinhoven et al. (2017) and Tran et al. (2020) reported common latent structures for the Big Five personality traits and the facets of the FFMQ, which broadly recovered the Big Five. Personality explained $46 \%$ of the mindfulness facets variance and most of their associations with mental health (Tran et al. 2020). While emotion regulation certainly can be labeled a mechanism/faculty of mindfulness, it thus appears not specific for mindfulness. This needs to be considered when assessing the associations of self-reported mindfulness with mental health and in treatment research. Also, future research should investigate selfreported mindfulness both vis-á-vis proposed mechanisms and personality traits like neuroticism.

\section{Nonattachment}

Nonattachment was previously described as a mindfulnessrelated construct that is empirically distinguishable from the mindfulness facets (Sahdra et al. 2016). In the present study, nonattachment was found to load diffusely on more than one factor, which leads to a different conclusion. It appears that nonattachment is neither fully distinct from the FFMQ facets (as it loaded on the same factors), nor a unitary mechanism/faculty of mindfulness on its own (as it loaded on multiple factors). However, the current study was based on the full NAS, rather than the abridged NAS-7 (see Sahdra et al. 2016), to retain comparability with some previous studies, which also have used the full NAS (e.g., Burzler et al. 2019; Tran et al. 2014). Clearly, nonattachment deserves further investigation in the future. It bears resemblance to the concept of cognitive defusion in acceptance and commitment therapy (Hayes et al. 1999) and shows incremental validity over the FFMQ in predicting psychological symptoms (Burzler et al. 2019) and satisfaction with life and life effectiveness (Sahdra et al. 2016).

\section{Attentional Control and Body Awareness}

Factors 1 and 2 concerned aspects of Attentional Control ("how") and Body Awareness ("what"), respectively. In the current study, these factors appeared to be more specific of meditation experience than Factors 4 and 5: Attentional Control for theoretical reasons (e.g., Hölzel et al. 2011; Kabat-Zinn 2015) and Body Awareness also for empirical reasons (largest mean difference between regular meditators and non-meditators in the current study). Observing, which loaded on body awareness (Factor 2, "what"), is also the one mindfulness facet that is most likely to increase with mindfulness practice (Baer et al. 2006).
Despite this, associations with mental health were small and mostly negligible for these two factors and - corroborating previous results (e.g., Baer et al. 2006) - in the opposite direction for body awareness (i.e., higher body awareness predicted lower mental health).

An association of Observing with lower mental health has been reported previously for non-meditators, but not meditators (e.g., Baer et al. 2006, 2008; Tran et al. 2013, 2014). In the current study, body awareness (Factor 2, which includes Observing) and emotion regulation (Factor 4, which includes an accepting attitude; "how") were uncorrelated among regular meditators and non-meditators alike. Meditators in the current study practiced mostly yoga and had less years of meditation experience than meditators in previous studies $(M d n=3$ vs. 8 years; see Tran et al. 2014). Also, yoga, in its Western, postural, and gymnastic (asana-based) style, might lack the meditative practice needed to foster an accepting attitude, especially if not trained for a long enough time.

Siegling and Petrides (2016) argued that Observing and its underlying factor should be omitted from the assessment of mindfulness in non-meditating samples. The current results add that one might skip the assessment of Attentional Control in non-meditating samples as well. Both factors, Attentional Control and Body Awareness, apparently did not capture relevant beneficial associations of mindfulness with mental health. Yet, both factors (together with Factor 3, see above) might be interesting for comparisons of meditators and non-meditators and might help in distinguishing between different types of meditation empirically (e.g., focused attention vs. open-monitoring meditation; Lutz et al. 2008). Thus, we suggest assessing these two factors specifically in research on meditation styles, but not necessarily in investigations of dispositional mindfulness.

\section{Assessing the Mechanisms/Faculties of Mindfulness}

None of the FFMQ facets loaded highest on any of the five factors. This suggests that even though the FFMQ apparently loaded on the common five underlying factors, other measures might do so with higher validity. Mindfulness scales, including the FFMQ, have been criticized for their complicated item formulations (Bergomi et al. 2013), and there is evidence that meditation experience affects how the items of the FFMQ are understood (Van Dam et al. 2009; note that strict measurement invariance related to scale and subscale scores, not items, in the current study). Items of some of the marker scales of the five common factors in the current study (i.e., EC Attentional Control, SBC Body Awareness, EQ Distanced Perspective, DERS Emotion Regulation Strategies, SBC Body Association) apparently addressed the same contents but appeared to be more clearly formulated (e.g., compare "I can observe unpleasant feelings without being drawn into them" [EQ subscale Distanced Perspective] with "Usually 
when I have distressing thoughts or images, I 'step back' and am aware of the thought or image without getting taken over by it" [FFMQ Nonreactivity]).

Similarity of content was also obvious (and demanded the modeling of correlated errors in the present study) for the items of the FFMQ facet Nonjudging and the (reversed) DERS subscale Acceptance of Emotional Responses, but further also, for example, for items of the FFMQ Observing facet and the (reversed) SBC subscale Body Awareness, and items of the FFMQ Describing facet and the (reversed) SBC subscale Body Association. Similarity of item content of these and other scales demands further attention in future research.

Based on the current results, future research should thus explore the utility of other measures to assess the mechanisms/faculties of mindfulness, rather than solely using dedicated measures such as the FFMQ or the scales it was derived from. This is backed by the observation that the marker scales also accounted for more variance of the examined mental health outcomes than did the FFMQ facets - not only in the current study but also compared with previous studies (Tran et al. 2014). Further, the use of alternative, and empirically validated, scales may make the links and conceptual overlaps of self-reported mindfulness with the constructs of other research fields more visible to researchers and practitioners. This may help in both guarding against conceptual confusion and strengthen the profile of mindfulness. Additionally, researchers should consider utilizing behavioral measures of mindfulness as well (e.g., Levinson et al. 2014).

\section{Limitations and Future Research}

Strengths of the present study include its application of ESEM, the large sample sizes, the conducted group comparisons, the detailed analysis of subscales, and the consideration of individual psychological symptoms as outcome variables instead of overall symptom scores. This level of detail allowed pointing out several specific associations between the mindfulness dimensions with specific groups and psychological symptoms.

Limitations concern the operationalization of mindfulness as a trait rather than a state (cf. Tanay and Bernstein 2013). Trait and state mindfulness may exhibit differential associations with the proposed mechanisms of mindfulness. Also, detailed analyses on the item level of both the mindfulness and mechanism measures were beyond the scope of this study. Our analysis is informative with regard to scale and subscale scores (which are mostly used in applied research), but not the item level. Even though reliability was mostly high (see Supplemental Materials), we cannot rule out that some of the utilized scales and subscales may have departed from unidimensionality or contained items with weak psychometric properties. This may be specifically true for the NAS. Yet, in addition to stated reasons of retaining comparability with some previously published studies, we also refrained from using the abridged NAS-7 as it had a lower reliability (.82 vs. .92) than the full scale in the current study.

Regular meditators and non-meditators were distinguished only according to their self-reported frequency of practice which may be prone to error. Furthermore, the use of alternative indices and cutoffs for testing measurement invariance may have changed the results of the present study somewhat. Finally, a cross-sectional design, as in the present study, appears to be adequate for the investigation of the associations of the proposed mechanisms of mindfulness with psychological outcomes. However, future studies could take advantage of a longitudinal design to allow for causal interpretations.

Future studies should also focus on specific meditation styles and standardized interventions (Van Dam et al. 2018), as they could be associated with different mechanisms of change. The current sample of regular mediators was relatively large, but groups were too small to investigate differences between specific meditation styles. Also, item level analyses and behavioral measures of mindfulness and neuroimaging methods may need to be included in future studies to assess the generalizability of the present results across modalities other than self-report and to control for the psychometric properties of self-report measures also on the item level. In addition, future studies should also take a closer look on the semantic overlap of the utilized measures (e.g., by using natural language processing) and build on our more exploratory results with stricter confirmatory approaches. Nonetheless, the results of the present study may guide future studies in developing new interventions and improving therapeutic effects.

Author Contributions KB collaborated with the design and executed the study, analyzed the data, and wrote the first draft of the paper. MV collaborated with the writing of the study. UST designed and supervised the study, provided the data, collaborated with the data analyses, and collaborated with the writing of the study. Kathrin Bednar and Ulrich S. Tran contributed equally to this work. All authors approved the final version of the manuscript for submission.

Funding Open access funding provided by University of Vienna.

\section{Compliance with Ethical Standards}

All procedures performed in this study adhere to the ethical standards of the 1964 Helsinki Declaration and with institutional guidelines of the School of Psychology, University of Vienna. Study participation did not affect the physical or psychological integrity, the right for privacy, or other personal rights or interests of the participants. Such being the case, according to national laws (Austrian Universities Act 2002), this study was exempted from formal ethical approval.

Conflict of Interest The authors declare that they have no conflict of interest.

Informed Consent Informed consent was obtained from all individual participants included in the study. 
Open Access This article is licensed under a Creative Commons Attribution 4.0 International License, which permits use, sharing, adaptation, distribution and reproduction in any medium or format, as long as you give appropriate credit to the original author(s) and the source, provide a link to the Creative Commons licence, and indicate if changes were made. The images or other third party material in this article are included in the article's Creative Commons licence, unless indicated otherwise in a credit line to the material. If material is not included in the article's Creative Commons licence and your intended use is not permitted by statutory regulation or exceeds the permitted use, you will need to obtain permission directly from the copyright holder. To view a copy of this licence, visit http://creativecommons.org/licenses/by/4.0/.

\section{References}

Annells, S., Kho, K., \& Bridge, P. (2016). Meditate don't medicate: how medical imaging evidence supports the role of meditation in the treatment of depression. Radiography, 22(1), 54-58. https://doi. org/10.1016/j.radi.2015.08.002.

Baer, R. A., Smith, G. T., \& Allen, K. B. (2004). Assessment of mindfulness by self-report: the Kentucky Inventory of Mindfulness Skills. Assessment, 11(3), 191-206. https://doi.org/10.1177/ 1073191104268029.

Baer, R. A., Smith, G. T., Hopkins, J., Krietemeyer, J., \& Toney, L. (2006). Using self-report assessment methods to explore facets of mindfulness. Assessment, 13(1), 27-45. https://doi.org/10.1177/ 1073191105283504.

Baer, R. A., Smith, G. T., Lykins, E., Button, D., Krietemeyer, J., Sauer, S., Walsh, E., Duggan, D., \& Williams, J. M. G. (2008). Construct validity of the Five Facet Mindfulness Questionnaire in meditating and nonmeditating samples. Assessment, 15(3), 329-342. https:// doi.org/10.1177/1073191107313003.

Behling, O., \& Law, K. S. (2000). Translating questionnaires and other research instruments: problems and solutions. Thousand Oaks: Sage.

Bergomi, C., Tschacher, W., \& Kupper, Z. (2013). The assessment of mindfulness with self-report measures: existing scales and open issues. Mindfulness, 4(3), 191-202. https://doi.org/10.1007/s12671012-0110-9.

Bieling, P. J., Hawley, L. L., Bloch, R. T., Corcoran, K. M., Levitan, R. D., Young, L. T., MacQueen, G. M., \& Segal, Z. V. (2012). Treatment-specific changes in decentering following mindfulnessbased cognitive therapy versus antidepressant medication or placebo for prevention of depressive relapse. Journal of Consulting and Clinical Psychology, 80(3), 365-372. https://doi.org/10.1037/ a0027483.

Bishop, S. R., Lau, M., Shapiro, S., Carlson, L., Anderson, N. D., Carmody, J., Segal, Z. V., Abbey, S., Speca, M., Velting, D., \& Devins, G. (2004). Mindfulness: a proposed operational definition. Clinical Psychology: Science and Practice, 11(3), 230-241. https:// doi.org/10.1093/clipsy/bph077.

Booth, T., \& Hughes, D. J. (2014). Exploratory structural equation modeling of personality data. Assessment, 21(3), 260-271. https:// doi.org/10.1177/1073191114528029.

Brown, K. W., Ryan, R. M., \& Creswell, J. D. (2007). Mindfulness: theoretical foundations and evidence for its salutary effects. Psychological Inquiry, 18(4), 211-237. https://doi.org/10.1080/ 10478400701598298.

Burzler, M. A., Voracek, M., Hos, M., \& Tran, U. S. (2019). Mechanisms of mindfulness in the general population. Mindfulness, 10(3), 469 480. https://doi.org/10.1007/s12671-018-0988-y.
Chiesa, A., Calati, R., \& Serretti, A. (2011). Does mindfulness training improve cognitive abilities? A systematic review of neuropsychological findings. Clinical Psychology Review, 31(3), 449-464. https://doi.org/10.1016/j.cpr.2010.11.003.

Chiesa, A., Serretti, A., \& Jakobsen, J. C. (2013). Mindfulness: top-down or bottom-up emotion regulation strategy? Clinical Psychology Review, 33(1), 82-96. https://doi.org/10.1016/j.cpr.2012.10.006.

Creswell, J. D., \& Lindsay, E. K. (2014). How does mindfulness training affect health? A mindfulness stress buffering account. Current Directions in Psychological Science, 23(6), 401-407. https://doi. org/10.1177/0963721414547415.

Delgado-Pastor, L. C., Ciria, L. F., Blanca, B., Mata, J. L., Vera, M. N., \& Vila, J. (2015). Dissociation between the cognitive and interoceptive components of mindfulness in the treatment of chronic worry. Journal of Behavior Therapy and Experimental Psychiatry, 48(3), 192-199. https://doi.org/10.1016/j.jbtep.2015.04.001.

Ehring, T., Fischer, S., Schnülle, J., Bösterling, A., \& Tuschen-Caffier, B. (2008). Characteristics of emotion regulation in recovered depressed versus never depressed individuals. Personality and Individual Differences, 44(7), 1574-1584. https://doi.org/10.1016/j.paid. 2008.01.013.

Fliege, H., Rose, M., Arck, P., Walter, O. B., Kocalevent, R.-D., Weber, C., \& Klapp, B. F. (2005). The perceived stress questionnaire (PSQ) reconsidered: validation and reference values from different clinical and healthy adult samples. Psychosomatic Medicine, 67(1), 78-88. https://doi.org/10.1097/01.psy.0000151491.80178.78.

Franke, G. H. (2016). Mini-SCL: German manual of the Mini Symptom Checklist [German]. Göttingen: Hogrefe.

Fresco, D. M., Moore, M. T., van Dulmen, M. H. M., Segal, Z. V., Ma, S. H., Teasdale, J. D., \& Williams, J. M. G. (2007). Initial psychometric properties of the Experiences Questionnaire: Validation of a selfreport measure of decentering. Behavior Therapy, 38(3), 234-246. https://doi.org/10.1016/j.beth.2006.08.003.

Freudenthaler, L., Turba, J. D., \& Tran, U. S. (2017). Emotion regulation mediates the associations of mindfulness on symptoms of depression and anxiety in the general population. Mindfulness, 8(5), 13391344. https://doi.org/10.1007/s12671-017-0709-y.

Gao, L., Curtiss, J., Liu, X., \& Hofmann, S. G. (2018). Differential treatment mechanisms in mindfulness meditation and progressive muscle relaxation. Mindfulness, 9(4), 1268-1279. https://doi.org/10. 1007/s12671-017-0869-9.

Gecht, J., Kessel, R., Mainz, V., Gauggel, S., Drueke, B., Scherer, A., \& Forkmann, T. (2014). Measuring decentering in self-reports: psychometric properties of the Experiences Questionnaire in a German sample. Psychotherapy Research, 24(1), 67-79. https:// doi.org/10.1080/10503307.2013.821635.

Giluk, T. L. (2009). Mindfulness, Big Five personality, and affect: a meta-analysis. Personality and Individual Differences, 47(8), 805811. https://doi.org/10.1016/j.paid.2009.06.026.

Goldberg, S. B., Tucker, R. P., Greene, P. A., Simpson, T. L., Hoyt, W. T., Kearney, D. J., \& Davidson, R. J. (2019). What can we learn from randomized clinical trials about the construct validity of self-report measures of mindfulness? A meta-analysis. Mindfulness, 10(5), 775-785. https://doi.org/10.1007/s12671018-1032-y.

Gratz, K. L., \& Roemer, L. (2004). Multidimensional assessment of emotion regulation and dysregulation: development, factor structure, and initial validation of the Difficulties in Emotion Regulation Scale. Journal of Psychopathology and Behavioral Assessment, 26(1), 41-54. https://doi.org/10.1023/B:JOBA. 0000007455.08539 .94 .

Greeson, J. M., Juberg, M. K., Maytan, M., James, K., \& Rogers, H. (2014). A randomized controlled trial of Koru: a mindfulness program for college students and other emerging adults. Journal of American College Health, 62(4), 222-233. https://doi.org/10.1080/ 07448481.2014 .887571 . 
Gu, J., Strauss, C., Bond, R., \& Cavanagh, K. (2015). How do mindfulness-based cognitive therapy and mindfulness-based stress reduction improve mental health and wellbeing? A systematic review and meta-analysis of mediation studies. Clinical Psychology Review, 37(4), 1-12. https://doi.org/10.1016/j.cpr. 2015.01.006.

Hayes, S. C., Strosahl, K. D., \& Wilson, K. G. (1999). Acceptance and commitment therapy: an experiential approach to behavior change. New York: Guilford.

Hodgins, H. S., \& Adair, K. C. (2010). Attentional processes and meditation. Consciousness and Cognition, 19(4), 872-878. https://doi. org/10.1016/j.concog.2010.04.002.

Hofmann, S. G., Sawyer, A. T., Witt, A. A., \& Oh, D. (2010). The effect of mindfulness-based therapy on anxiety and depression: a metaanalytic review. Journal of Consulting and Clinical Psychology, 78(2), 169-183. https://doi.org/10.1037/a0018555.

Hölzel, B. K., Lazar, S. W., Gard, T., Schuman-Olivier, Z., Vago, D. R., \& Ott, U. (2011). How does mindfulness meditation work? Proposing mechanisms of action from a conceptual and neural perspective. Perspectives on Psychological Science, 6(6), 537-559. https://doi.org/10.1177/1745691611419671.

Hu, L. T., \& Bentler, P. M. (1999). Cutoff criteria for fit indexes in covariance structure analysis: conventional criteria versus new alternatives. Structural Equation Modeling, 6(1), 1-55. https://doi.org/ 10.1080/10705519909540118.

Kabat-Zinn, J. (2005). Full catastrophe living: using the wisdom of your body and mind to face stress, pain, and illness. New York: Delta trade.

Kabat-Zinn, J. (2015). Mindfulness. Mindfulness., 6(6), 1481-1483. https://doi.org/10.1007/s12671-015-0456-X.

Keng, S.-L., Smoski, M. J., \& Robins, C. J. (2011). Effects of mindfulness on psychological health: a review of empirical studies. Clinical Psychology Review, 31(6), 1041-1056. https://doi.org/10.1016/j. cpr.2011.04.006.

Khoury, B., Lecomte, T., Fortin, G., Masse, M., Therien, P., Bouchard, V., Chapleau, M.-A., Paquin, K., \& Hofmann, S. G. (2013). Mindfulness-based therapy: a comprehensive meta-analysis. Clinical Psychology Review, 33(6), 763-771. https://doi.org/10. 1016/j.cpr.2013.05.005.

Lamis, D., \& Dvorak, R. D. (2014). Mindfulness, nonattachment, and suicide rumination in college students: the mediating role of depressive symptoms. Mindfulness, 5(5), 487-496. https://doi.org/10. 1007/s12671-013-0203-0.

Lao, S.-A., Kissane, D., \& Meadows, G. (2016). Cognitive effects of MBSR/MBCT: a systematic review of neuropsychological outcomes. Consciousness and Cognition, 45, 109-123. https://doi. org/10.1016/j.concog.2016.08.017.

Levinson, D. B., Stoll, E. L., Kindy, S. D., Merry, H. L., \& Davidson, R. J. (2014). A mind you can count on: validating breath counting as a behavioral measure of mindfulness. Frontiers in Psychology, 5, article 1202. https://doi.org/10.3389/fpsyg.2014.01202.

Linehan, M. (2015). DBT skills training handouts and worksheets (2nd ed.). New York, NY: Guilford.

Marsh, H. W., Morin, A. J. S., Parker, P. D., \& Kaur, G. (2014). Exploratory structural equation modeling: an integration of the best features of exploratory and confirmatory factor analysis. Annual Review of Clinical Psychology, 10, 85-110. https://doi.org/10. 1146/annurev-clinpsy-032813-153700.

Mehling, W. E., Wrubel, J., Daubenmier, J. J., Price, C. J., Kerr, C. E., Silow, T., Gopisetty, V., \& Stewart, A. L. (2011). Body awareness: a phenomenological inquiry into the common ground of mind-body therapies. Philosophy, Ethics, and Humanities in Medicine, 6, article 6. https://doi.org/10.1186/1747-5341-6-6.

Muthén, L. K., \& Muthén, B. O. (1998-2017). Mplus user's guide (8th ed.). Los Angeles: Muthén \& Muthén.

Park, T., Reilly-Spong, M., \& Gross, C. R. (2013). Mindfulness: a systematic review of instruments to measure an emergent patientreported outcome (PRO). Quality of Life Research, 22(4), 26392659. https://doi.org/10.1007/s11136-013-0395-8.

Price, C. J., \& Thompson, E. A. (2007). Measuring dimensions of body connection: body awareness and bodily dissociation. Journal of Alternative and Complementary Medicine, 13(9), 945-953. https:// doi.org/10.1089/acm.2007.0537.

Putnick, D. L., \& Bornstein, M. H. (2016). Measurement invariance conventions and reporting: the state of the art and future directions for psychological research. Developmental Review, 41, 71-90. https://doi.org/10.1016/j.dr.2016.06.004.

Quezada-Berumen, L. d. C., González-Ramírez, M. T., Cebolla, A., Soler, J., \& Garcia-Campayo, J. (2014). Body awareness and mindfulness: validation of the Spanish version of the Scale of Body Connection. Actas Españolas de Psiquiatría, 42(2), 5767.

Revelle, W. (2008). Psych: procedures for personality and psychological research ( $\mathrm{R}$ package version 1.8.12).

Sahdra, B. K., Shaver, P. R., \& Brown, K. W. (2010). A scale to measure nonattachment: a Buddhist complement to Western research on attachment and adaptive functioning. Journal of Personality Assessment, 92(2), 116-127. https://doi.org/10.1080/ 00223890903425960.

Sahdra, B., Ciarrochi, J., \& Parker, P. (2016). Nonattachment and mindfulness: related but distinct constructs. Psychological Assessment, 28(7), 819-829. https://doi.org/10.1037/pas0000264.

Segal, Z. V., Williams, J. M. G., \& Teasdale, J. D. (2013). Mindfulnessbased cognitive therapy for depression (2nd ed.). New York: Guilford.

Servaas, M. N., van der Velde, J., Costafreda, S. G., Horton, P., Ormel, J., Riese, H., \& Aleman, A. (2013). Neuroticism and the brain: a quantitative meta-analysis of neuroimaging studies investigating emotion processing. Neuroscience and Biobehavioral Reviews, 37(8), 1518-1529. https://doi.org/10. 1016/j.neubiorev.2013.05.005.

Shapiro, S. L., Carlson, L. E., Astin, J. A., \& Freedman, B. (2006). Mechanisms of mindfulness. Journal of Clinical Psychology, 62(3), 373-386. https://doi.org/10.1002/jclp.20237.

Siegling, A. B., \& Petrides, K. V. (2016). Zeroing in on mindfulness facets: similarities, validity, and dimensionality across three independent measures. PLOS ONE, 11, article e0153073. https://doi. org/10.1371/journal.pone.0153073.

Spinhoven, P., Huijbers, M. J., Zheng, Y., Ormel, J., \& Speckens, A. E. M. (2017). Mindfulness facets and Big Five personality facets in persons with recurrent depression in remission. Personality and Individual Differences, 110, 109-114. https://doi.org/10.1016/j. paid.2017.01.045.

Tanay, G., \& Bernstein, A. (2013). State mindfulness scale (SMS): development and initial validation. Psychological Assessment, 25(4), 1286-1299. https://doi.org/10.1037/a0034044.

Tran, U. S., Glück, T. M., \& Nader, I. W. (2013). Investigating the five facet mindfulness questionnaire (FFMQ): construction of a short form and evidence of a two-factor higher order structure of mindfulness. Journal of Clinical Psychology, 69(9), 951-965. https://doi. org/10.1002/jclp.21996.

Tran, U. S., Cebolla, A., Glück, T. M., Soler, J., Garcia-Campayo, J., \& von Moy, T. (2014). The serenity of the meditating mind: 
a cross-cultural psychometric study on a two-factor higher order structure of mindfulness, its effects, and mechanisms related to mental health among experienced meditators. PLOS ONE, 9, article e110192. https://doi.org/10.1371/journal.pone. 0110192

Tran, U. S., Wasserbauer, J., \& Voracek, M. (2020). Testing the incremental validity of dispositional mindfulness over and above the Big Five in accounting for mental health: a facetlevel structural-equation modeling and predictor communality and dominance approach. Personality and Individual Differences, 156, article 109769. https://doi.org/10.1016/j. paid.2019.109769.

Van Dam, N. T., Earleywine, M., \& Danoff-Burg, S. (2009). Differential item function across meditators and non-meditators on the Five Facet Mindfulness Questionnaire. Personality and Individual Differences, 47(5), 516-521. https://doi.org/10.1016/j.paid.2009. 05.005 .

Van Dam, N. T., van Vugt, M. K., Vago, D. R., Schmalzl, L., Saron, C. D., Olendzki, A., Meissner, T., Lazar, S. W., Kerr, C. E., Gorchov, J., Fox, K. C. R., Field, B. A., Britton, W. B.,
Brefczynski-Lewis, J. A., \& Meyer, D. E. (2018). Mind the hype: a critical evaluation and prescriptive agenda for research on mindfulness and meditation. Perspectives on Psychological Science, 13(1), 36-61. https://doi.org/10.1177/ 1745691617709589 .

van der Velden, A. M., Kuyken, W., Wattar, U., Crane, C., Pallesen, K. J., Dahlgaard, J., Fjorback, L. O., \& Piet, J. (2015). A systematic review of mechanisms of change in mindfulness-based cognitive therapy in the treatment of recurrent major depressive disorder. Clinical Psychology Review, 37(4), 26-39. https://doi.org/10.1016/j.cpr. 2015.02.001.

Wiltink, J., Vogelsang, U., \& Beutel, M. E. (2006). Temperament and personality: The German version of the Adult Temperament Questionnaire (ATQ). GMS Psycho-Social-Medicine, 3, Article Doc10.

Publisher's Note Springer Nature remains neutral with regard to jurisdictional claims in published maps and institutional affiliations. 